\title{
The Effect of Modelling Parameters on the Value of GMWB Guarantees
}

\author{
Z. Chen, \\ K. Vetzal ${ }^{\dagger}$ \\ P.A. Forsyth ${ }^{\ddagger}$
}

December 17, 2007

\begin{abstract}
In this article, an extensive study of the no-arbitrage fee for Guaranteed Minimum Withdrawal Benefit (GMWB) variable annuity riders is carried out. Taking into account such contractual features as the separation of mutual fund fees and the fees earmarked for hedging the guarantee, as well as the possibility of jumps in the value of the underlying asset, increases the value of the GMWB guarantee considerably. We also explore the effects of various modelling assumptions on the optimal withdrawal strategy of the contract holder, as well as the impact on the guarantee value of sub-optimal withdrawal behaviour. Our general conclusions are that only if several unrealistic modelling assumptions are made is it possible to obtain GMWB fees in the same range as is normally charged. In all other cases, it would appear that typical fees are not enough to cover the cost of hedging these guarantees.
\end{abstract}

Keywords: Variable annuities, pensions, GMWB guarantees

Insurance Branch Code: IM10;IE43;IB13

JEL Classification: G22

\section{Introduction}

Throughout the developed world, both companies and governments are moving away from defined benefit pension plans to defined contribution (DC) plans. Under a DC plan, the holder has control of the accumulated pension assets, but assumes the risk of investing these assets. Most DC holders have no choice but to invest in risky assets in order to provide sufficient income during their retirement years.

As pointed out in Milevsky and Salisbury (2006), before retiring, the DC plan holder is exposed to market risks, but the order of random returns is immaterial. However, once the DC plan holder retires, and begins to withdraw funds from the plan, the holder is also exposed to the risk from the

\footnotetext{
*David R. Cheriton School of Computer Science, University of Waterloo, Waterloo ON, Canada N2L 3G1 e-mail: z4chen@uwaterloo.ca

${ }^{\dagger}$ School of Accounting and Finance, University of Waterloo, Waterloo ON, Canada N2L 3G1 kvetzal@uwaterloo.ca

${ }^{\ddagger}$ David R. Cheriton School of Computer Science, University of Waterloo, Waterloo ON, Canada N2L 3G1 paforsyt@uwaterloo.ca
} 
order of the returns. Losses in the early years of retirement can be devastating, and can result in a rapid exhaustion of the plan.

In order to the manage the risk associated with the random order of returns after the DC holder retires and begins to withdraw funds from the plan, many insurance companies are offering variable annuities with a Guaranteed Minimum Withdrawal Benefit (GMWB) rider.

A GMWB contract involves an initial lump sum payment to an insurance company. These funds are then invested in risky assets, typically a mutual fund. The policy holder is then entitled to withdraw funds (usually annually or semi-annually) at a contractually specified rate. The GMWB promises to return at least the entire original investment, regardless of the performance of the underlying risky investment. The policy holder may withdraw at a higher or lower rate than the contract rate (or even to surrender instantaneously) subject to certain penalties and conditions.

In this article, we will value the GMWB contract from a financial no-arbitrage point of view. Previous work on GMWBs following this approach includes Milevsky and Salisbury (2006), Dai et al. (2007), and Bauer et al. (2006). In Milevsky and Salisbury (2006) and Dai et al. (2007), an idealized contract is studied and valued using optimal stochastic control techniques. More general contracts are considered in Bauer et al. (2006). All of these authors agree that GMWB guarantees appear to be underpriced in the market. In other words, insurance companies do not appear to be collecting enough in the way of fees to cover all of the costs associated with hedging these guarantees.

The objective of this paper is to examine in some detail some generalizations of the idealized contract discussed in Dai et al. (2007). We will determine the effect of various parameters and assumptions on the fair fee associated with the guarantee. In particular, we study the following:

- In practice, the underlying mutual fund charges a separate layer of fees for managing the fund. It has been suggested that the apparent underfunding of the GMWB rider can be explained if we assume that some of these mutual fund fees are diverted to managing the GMWB rider. However, our experience in pricing other pension plan guarantees (Windcliff et al., 2001, 2002; Le Roux, 2000) suggests that the mutual fund fees are not available for hedging purposes. We will derive the no-arbitrage partial differential equation (PDE) which results from this fee splitting, and provide numerical results which show the effect of the fee splitting. This fee separation is important in practice, and does not appear to have been taken into account in previous work (e.g. Milevsky and Salisbury, 2006; Dai et al., 2007; Bauer et al., 2006). Inclusion of this fee separation increases the value of the GMWB rider.

- Cramer et al. (2007) discuss various assumptions about investor behavior when pricing variable annuities. A conservative approach is to assume optimal investor behavior, and then to recognize extraordinary earnings in the event of sub-optimal behavior. Another possibility is to develop a model of non-optimal behavior, and incorporate this into the pricing model. We will examine both approaches in this paper. Our base case assumes optimal behaviour, but we also model the effect of sub-optimal withdrawal behavior using the approach suggested in Ho et al. (2005). Sub-optimal behavior considerably reduces the value of the GMWB rider.

- We will include results with both the classic Geometric Brownian motion process for the underlying asset, as well as a jump diffusion process, which may be a more realistic model for long term guarantees. Making the assumption that there is reasonable (risk neutral) probability of a market crash during the lifetime of the guarantee dramatically increases the value of the GMWB rider. 
- We will also examine the effect of various contract parameters, such as reset provisions, maturities, withdrawal intervals, and surrender charges. Some contract features (e.g. the reset provision) have almost no effect on the value of the guarantee, while others have considerable influence. In addition to the value of the guarantee, we explore the impact of some of these various alternative modelling assumptions on the policyholder's optimal withdrawal strategy. Plots of the contour levels of the optimal withdrawal amounts show that the investor's optimal strategy can be quite sensitive to modelling assumptions.

\section{Contract Description}

We briefly review the GMWB rider contract description. For more details, we refer the reader to Milevsky and Salisbury (2006) and Dai et al. (2007). The contract consists of a personal subaccount invested in a risky asset, as well as a guarantee account. At the inception of the contract, the holder pays a lump sum to the insurer, which forms the initial balance of the sub-account and the guarantee account. The policy holder pays a fee proportional to the sub-account balance.

The GMWB rider allows the holder to withdraw funds on an annual or semi-annual basis. Each withdrawal reduces the amount in the guarantee account and the sub-account on a dollar for dollar basis. The holder can continue to withdraw as long as the guarantee account is above zero, even if the sub-account balance is zero. At the maturity of the contract, the holder receives the maximum of the sub-account balance or the guarantee account balance, net of any fees.

Note that this represents an idealized GMWB rider, and there are many variations of this basic contract.

\section{Formulation}

We assume that the withdrawal occurs only at predetermined discrete times. This problem can also be posed in terms of continuous withdrawals (Chen and Forsyth, 2007). However, as we shall verify in some numerical tests, the value of the continuous withdrawal formulation is very close to the discrete withdrawal case if the withdrawal intervals are less than one year. In addition, many contracts only allow discrete withdrawals.

Similar to the work in Milevsky and Salisbury (2006) and Dai et al. (2007), we will ignore mortality effects in the following. We plan to study the effect of mortality contract features in future work.

Let $W$ denote the balance of the personal variable annuity sub-account. Recall that $W$ is invested in a risky asset. Let $A$ denote the current balance of the guarantee account. Let $W_{0}$ be the initial sub-account balance and guarantee account balance which is the same as the premium paid upfront. Then $A$ can have any value lying in $\left[0, W_{0}\right]$. Let $\alpha_{t o t} \geq 0$ denote the proportional total fees (including mutual fund management expenses and fees charged for the GMWB rider) paid by the policy holder. Let $\alpha_{g}$ be the fee paid to fund the guarantee, and $\alpha_{m}$ be the mutual fund management fee, so that $\alpha_{t o t}=\alpha_{g}+\alpha_{m}$.

We assume that the risk neutral process of the sub-account value $W$ is modeled by a stochastic differential equation (SDE) given by

$$
\begin{aligned}
& d W=\left(r-\alpha_{t o t}\right) W d t+\sigma W d Z+d A, \quad \text { if } W>0 \\
& d W=0, \quad \text { if } W=0 .
\end{aligned}
$$




\begin{tabular}{cc}
\hline Year & Surrender Charge $\kappa$ \\
\hline $0 \leq t<2$ & $8 \%$ \\
$2 \leq t<3$ & $7 \%$ \\
$3 \leq t<4$ & $6 \%$ \\
$4 \leq t<5$ & $5 \%$ \\
$5 \leq t<6$ & $4 \%$ \\
$6 \leq t<7$ & $3 \%$ \\
$t \geq 7$ & $0 \%$ \\
\hline
\end{tabular}

TABLE 1: Time-dependent surrender charges $\kappa(t)$.

Let $\tau=T-t$, where $T$ is the expiry date of the contract. Let $\tau^{k}, k=0, \ldots, K-1$ be the $k$ th withdrawal time going backwards in time with $\tau^{0}=0$ and $\tau^{K}=T$. There is no withdrawal allowed at $\tau^{K}=T$ (the inception of the contract). We denote by $\gamma^{k}$ the control variable representing the discrete withdrawal amount at $\tau=\tau^{k} ; \gamma^{k}$ can take any value in $\gamma^{k} \in[0, A]$.

Let $G^{k}$ represent the contract withdrawal amount at $\tau^{k}$. If $\gamma^{k} \leq G^{k}$, no penalty is imposed. However, if $\gamma^{k}>G^{k}$, then there is a proportional penalty charge $\kappa\left(\gamma^{k}-G^{k}\right)$, that is, the net amount received by the policyholder is $\gamma^{k}-\kappa\left(\gamma^{k}-G^{k}\right)$ if $\gamma^{k}>G^{k}$, where $\kappa$ is a positive constant representing the deferred surrender charge. We assume that the penalty (surrender) fees are available to fund the GMWB guarantee.

Consequently, the cash-flow received by the policyholder at the discrete withdrawal time $\tau=\tau^{k}$ as a function of $\gamma^{k}$, denoted by $f\left(\gamma^{k}\right)$, is given by

$$
f\left(\gamma^{k}\right)=\left\{\begin{array}{ll}
\gamma^{k} & \text { if } 0 \leq \gamma^{k} \leq G^{k} \\
\gamma^{k}-\kappa\left(\gamma^{k}-G^{k}\right) & \text { if } \gamma^{k}>G^{k}
\end{array} .\right.
$$

In a typical contract, the deferred surrender charge $\kappa=\kappa(t)$ is time-dependent and normally decreases over time to zero. Table 1 shows a typical specification for $\kappa(t)$.

Let $V(W, A, \tau)$ denote the total value of the variable annuity including the GMWB guarantee as a function of $(W, A, \tau)$. Note that $V$ includes all the cash flows to the policy holder, not just the GMWB guarantee cash flows.

In Appendix A, we derive the equations which determine the no-arbitrage value of $V$. As in Dai et al. (2007), the terminal condition for the annuity is

$$
V(W, A, \tau=0)=\max (W, A(1-\kappa)) .
$$

At the withdrawal time $\tau=\tau^{k}, V$ satisfies the following optimality condition

$$
V\left(W, A, \tau^{k+}\right)=\sup _{\gamma^{k} \in[0, A]}\left[V\left(\max \left(W-\gamma^{k}, 0\right), A-\gamma^{k}, \tau^{k}\right)+f\left(\gamma^{k}\right)\right], \quad k=0, \ldots, K-1,
$$

where $\tau^{k+}$ denotes the time infinitesimally after $\tau^{k}$ (recall from the definition of $\tau$ that time is running backwards). Equation (5) has the intuitive interpretation that the policyholder will select the withdrawal which maximizes the total value of the cash flow received and the future value of the annuity after withdrawal. 


\begin{tabular}{clc}
\hline & \multicolumn{1}{c}{ Parameter } & Value \\
\hline$T$ & Expiry time & 10 years \\
$r$ & Interest rate & $5 \%$ \\
$G$ & Contract withdrawal amount & 10 \\
$W_{0}$ & Initial lump-sum premium & 100 \\
$\sigma$ & Volatility & .15 \\
$\Delta \tau^{k}$ & Withdrawal interval & 1 year \\
$\alpha_{m}$ & Mutual fund fee & $1 \%$ \\
\hline
\end{tabular}

TABLE 2: Base case parameters.

Within each time interval $\left[\tau^{k+}, \tau^{k+1}\right], k=0, \ldots, K-1$, the annuity value function $V(W, A, \tau)$, solves the following linear PDE (see Appendix A) which has $A$ dependence only through equation (5):

$$
V_{\tau}=\mathcal{L} V+\alpha_{m} W, \quad \tau \in\left[\tau^{k+}, \tau^{k+1}\right], \quad k=0, \ldots, K-1 .
$$

where the operator $\mathcal{L}$ is

$$
\mathcal{L} V=\frac{1}{2} \sigma^{2} W^{2} V_{W W}+\left(r-\alpha_{t o t}\right) W V_{W}-r V .
$$

The no-arbitrage insurance fee $\alpha_{g}$ (recall $\alpha_{t o t}=\alpha_{g}+\alpha_{m}$ ) is determined so that the contract value $V$ at $\tau=T$ is equal to the initial premium $W_{0}$ paid by the investor (Milevsky and Salisbury, 2006; Dai et al., 2007; Cramer et al., 2007). The equations (4-7) are solved numerically using the methods described in Chen and Forsyth (2007). In the following, all results are given correct to the number of digits shown, based on grid and timestep refinement studies.

\section{$4 \quad$ Numerical Results}

\subsection{Base Case}

We first compute the value of the guarantee for a representative base case, and then perturb the problem parameters and compare to this base case. The base case parameters are given in Table 2, with the surrender charge $\kappa(t)$ (see equation (3)) given in Table 1.

For this base case, the no-arbitrage insurance fee is $\alpha_{g}=117$ basis points. Figure 1 shows a contour plot of the optimal withdrawal strategy $\gamma^{k}$ at the first withdrawal time $(t=1)$ for different values of $W$ and $A$. In particular, we show contour levels of $\gamma=10, \gamma=.2$. We choose these two contour levels to show that in some cases the optimal withdrawal amount $\gamma$ rapidly changes from the contract amount $G^{k}$ to zero. (Due to contouring artifacts, a contour value $\gamma<.2$ results in very jagged contour levels, since it is difficult to determine numerically the zero withdrawal region). For practical purposes, the $\gamma=.2$ contour level shows the region where it is optimal to withdraw nothing. For a discussion of the conditions under which it may be optimal to withdraw nothing, see Chen and Forsyth (2007).

From Figure 1, we can observe the following:

- There is a shaded region in the left side of the figure representing excessive withdrawals (i.e. withdrawals above the contract amount) when $A$ dominates $W$. In this region, it is unlikely 


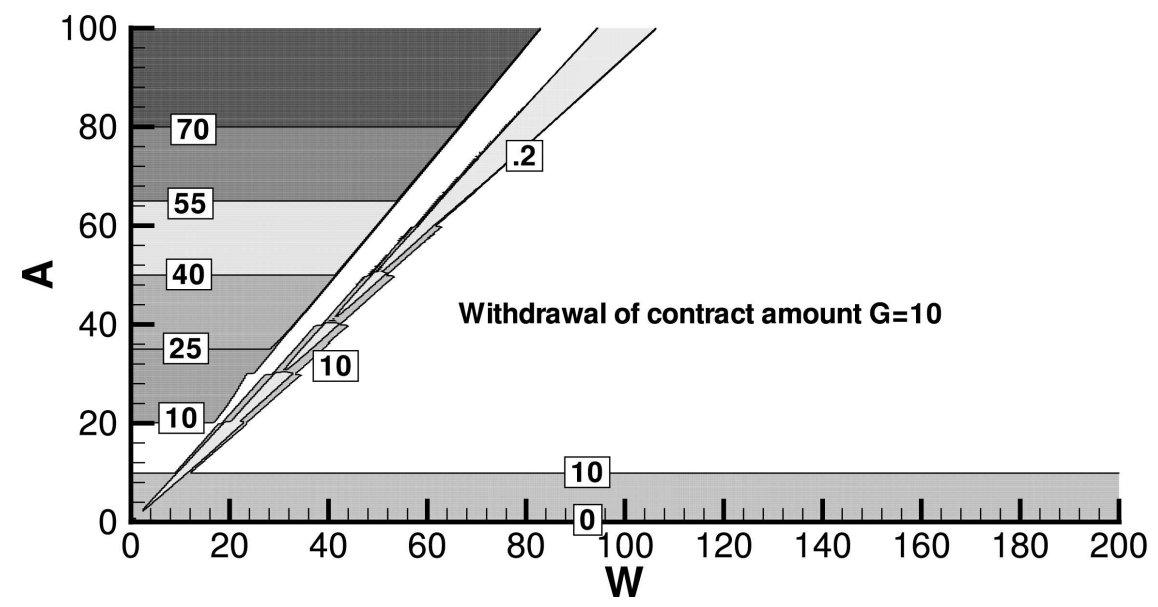

Figure 1: Optimal withdrawal strategy of the GMWB guarantee at the first withdrawal time $(t=1$ year) in the $(W, A)$-plane. Contour lines representing the same withdrawal levels are shown, where the withdrawal amounts are posted on those contour lines. Parameters for this example are given in Tables 1 and 2. The contour line showing $\gamma=.2$ shows the region where it is optimal to withdraw essentially nothing.

that the amount in the risky sub-account will ever exceed the guarantee account. Intuitively, the investor withdraws as rapidly as possible (subject to minimizing the surrender charges) because the total guarantee available is just $W_{0}$ and delaying withdrawal is costly due to a lower present value of the funds withdrawn. As a specific example, consider the case where $(W, A)=(0.0,80)$. From Table 1 , the investor will receive .92 of any withdrawal above 10. Also, note (from Table 2) that $r=.05$. In this case, it is optimal to withdraw 70 immediately, and then withdraw 10 the next year. The present value of this strategy is $10+60 \times .92+e^{-.05} 10=74.71$. This is slightly better than withdrawing 80 immediately, which has value of $10+70 \times .92=74.40$.

- There is a blank region in the right side of the figure representing the withdrawal of the contract amount $\gamma^{k}=G^{k}$.

- There is a narrow area surrounding the line $W=A$, in which the optimal strategy is to withdraw an amount less than $G^{k}$. The contour line for withdrawing $\gamma^{k}=.2$ is also shown, to illustrate the rapid change in the withdrawal amount over a small region in the $(W, A)$ plane.

\subsection{Effect of Volatility}

The insurance fees for different choices of volatility $\sigma$ are given in Table 3. The table shows that volatility has a large effect on the no-arbitrage value of the guarantee fee $\alpha_{g}$. For example, the fee level almost doubles when $\sigma$ is set to .20 compared to the base case of $\sigma=.15$.

Figures 2, 3 and 4 show the optimal withdrawal strategy for $\sigma=.20$ at the first, fourth and eighth withdrawal time forwards in time (with respect to $t=1$ st year, $t=4$ th year and $t=8$ th year). The figures reveal that:

- Compared with Figure 1, increasing volatility generates another excessive withdrawal region when $W$ dominates $A$. When $W \gg A$, the guarantee is effectively out the money. Hence the 


\begin{tabular}{cc}
\hline Volatility $\sigma$ & Insurance Fee $\alpha_{g}$ \\
\hline .15 & 117 b.p. \\
.20 & 214 b.p. \\
.25 & 326 b.p. \\
.30 & 440 b.p. \\
.35 & 552 b.p. \\
\hline
\end{tabular}

TABLE 3: GMWB guarantee fees $\alpha_{g}$ determined with different choices of the volatility $\sigma$. Other parameter values are given in Tables 1 and 2.

investor withdraws an amount which minimizes the fees charged for this out of the money guarantee, subject to minimizing the surrender charges.

- As $t$ increases, the shaded regions representing excessive withdrawals expand. At the same time, the blank region representing the withdrawal of the contract amount $G^{k}$ shrinks. This is due to the decrease of the surrender charge $\kappa$ over time, which imposes a smaller penalty on excessive withdrawals. However, it is also interesting to note that the no-withdrawal region (i.e. the region enclosed by the $\gamma=.2$ contour) expands as well.

- Excessive withdrawals at a later time (i.e. a larger $t$ ) will result in an equal or less remaining balance in the guarantee account compared with excessive withdrawals at an earlier time (i.e. a smaller $t$ ). In particular, at the eighth withdrawal time when $\kappa=0$, the optimal strategy is to withdraw the whole amount from the guarantee account in the left shaded region (where $A$ dominates $W$ ) as well as in the right shaded region (where $W$ dominates $A$ ), excluding the triangular area surrounding the line $W=A$. The area near $W=A$ can be regarded as an at the money put option. Since there are only two years left in the contract, the fees charged for this at the money put are comparatively low, and hence it is worthwhile for the policyholder to keep the option intact (i.e. not to withdraw).

\subsection{Incorporating Price Jumps}

Many studies have shown that for long term contingent claims, it is important to consider jump processes (Andersen and Andreasen, 2000, e.g.). For this example, we assume that the dynamics of $W$ follows a jump diffusion process given by

$$
\begin{aligned}
& d W=\left(r-\alpha_{t o t}-\lambda \beta\right) W d t+\sigma W d Z+(\eta-1) W d q+d A, \quad \text { if } W>0 \\
& d W=0, \quad \text { if } W=0,
\end{aligned}
$$

where:

- $d q$ is an independent Poisson process with $d q=\left\{\begin{array}{ll}0 & \text { with probability } 1-\lambda d t \\ 1 & \text { with probability } \lambda d t\end{array}\right.$,

- $\lambda$ is the jump intensity representing the mean arrival rate of the Poisson process, 


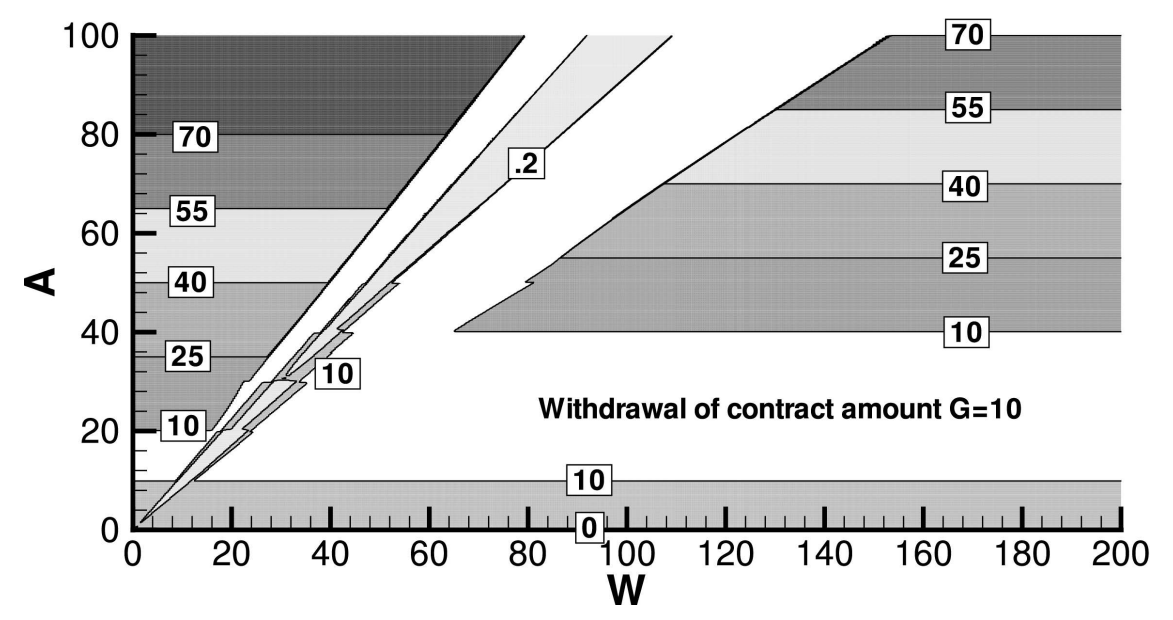

FIGURE 2: Optimal withdrawal strategy of the GMWB guarantee at the first withdrawal time forwards in time $(t=1$ year) in the $(W, A)$-plane with $\sigma=.20$. Other parameter values are given in Tables 1 and 2. The contour line showing $\gamma=.2$ shows the region where it is optimal to withdraw essentially nothing.

- $\eta$ is a random variable representing the jump size of $W$; we assume that $\eta$ follows a log-normal distribution $g(\eta)$ given by

$$
g(\eta)=\frac{1}{\sqrt{2 \pi} \zeta \eta} \exp \left(-\frac{(\log (\eta)-\nu)^{2}}{2 \zeta^{2}}\right)
$$

with parameters $\zeta$ and $\nu$,

- $\beta=E[\eta-1]$, where $E[\eta]=\exp \left(\nu+\zeta^{2} / 2\right)$ given the distribution function $g(\eta)$ in $(10)$.

Note that we are working here in an incomplete market, so that the equivalent martingale pricing measure is not in general unique. As in Andersen and Andreasen (2000), we calibrate the parameters of equation (8) to traded prices of options. This means that the parameters of (8) correspond to those from the market's pricing measure.

Using (8), it is straightforward to to generalize the pricing PDE (6) to the pricing partial integro differential equation (PIDE)

$$
V_{\tau}-\mathcal{L} V-\mathcal{H} V-\alpha_{m} W=0, \quad \tau \in\left[\tau^{k+}, \tau^{k+1}\right], \quad k=0, \ldots, K-1,
$$

where the operator $\mathcal{H}$ satisfies

$$
\begin{aligned}
\mathcal{H} V & =\lambda E\left[V(W \eta)-V-(\eta-1) W V_{W}\right] \\
& =\lambda \int_{0}^{\infty} V(W \eta) g(\eta) d \eta-\lambda V-\lambda \beta W V_{W}
\end{aligned}
$$

We solve PIDE (11) using the numerical methods described in d'Halluin et al. (2005). Table 4 gives the jump diffusion parameters which we will use in our tests. These parameters are essentially the (rounded) parameters obtained in Andersen and Andreasen (2000) from calibration to S\&P 500 index option prices. 


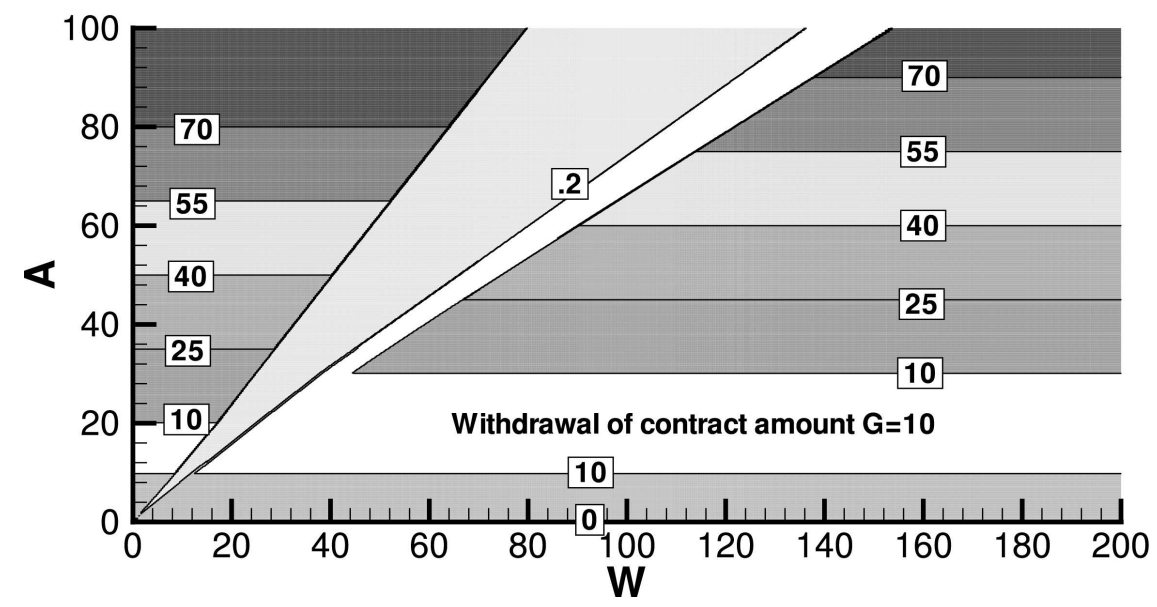

FIGURE 3: Optimal withdrawal strategy of the GMWB guarantee at the fourth withdrawal time forwards in time $(t=4$ years $)$ in the $(W, A)$-plane with $\sigma=.20$. Other parameter values are given in Tables 1 and 2. The contour line showing $\gamma=.2$ shows the region where it is optimal to withdraw essentially nothing.

\begin{tabular}{cl}
\hline Parameter & Value \\
\hline$\lambda$ & .1 \\
$\zeta$ & .45 \\
$\nu$ & -.9 \\
$\sigma$ & .15 \\
\hline
\end{tabular}

TABLE 4: Parameters for the jump diffusion case.

The fair insurance fees for the jump and no jump cases are given in Table 5 . The table shows that incorporating jumps greatly increases the insurance fees. Note that a volatility of $\sigma=.15$ may appear to be reasonable if one examines recent long term data for implied volatility of a major stock index. However, the implied volatilities are based on short term options, which do not capture long term information about jumps. Table 5 shows that ignoring the possibility of jumps for long term guarantees may severely underestimate the hedging cost. Methods for hedging contracts under jump diffusions are discussed in (He et al., 2006; Kennedy et al., 2006).

\subsection{Separation of Mutual Fund Fee}

Recall that in equation (6), we are careful to distinguish between the fees used to manage the underlying mutual fund $\left(\alpha_{m}\right)$ and the fees used to fund the GMWB guarantee $\left(\alpha_{g}\right)$. This is

\begin{tabular}{cc}
\hline & Insurance Fee $\alpha_{g}$ \\
\hline With jump & 356 b.p. \\
Base case (no jump) & 117 b.p. \\
\hline
\end{tabular}

TABle 5: Insurance fees with/without price jumps. Parameters are given in Tables 1, 2 and 4. 


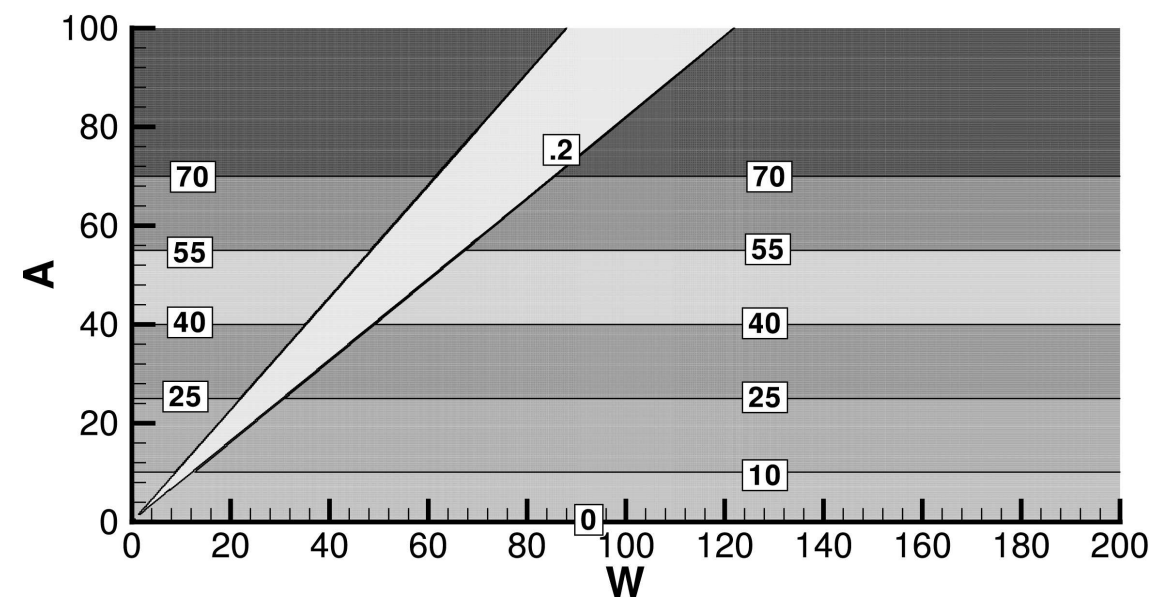

FIGURE 4: Optimal withdrawal strategy of the GMWB guarantee at the eighth withdrawal time forwards in time $(t=8$ years) in the $(W, A)$-plane with $\sigma=.20$. Other parameter values are given in Tables 1 and 2. The contour line showing $\gamma=.2$ shows the region where it is optimal to withdraw essentially nothing.

\begin{tabular}{cc}
\hline Mutual Fund Fee $\alpha_{m}$ & Insurance Fee $\alpha_{g}$ \\
\hline $0.0 \%$ & 88 b.p. \\
$0.5 \%$ & 102 b.p. \\
$1.0 \%$ & 117 b.p. \\
$1.5 \%$ & 136 b.p. \\
$2.0 \%$ & 157 b.p. \\
$2.5 \%$ & 184 b.p. \\
\hline
\end{tabular}

TABLE 6: Insurance fees determined by different choices of the mutual fund fees. Other parameter values are given in Tables 1 and 2.

because the provider of the GWMB rider may be a completely separate business unit from the unit managing the mutual fund (Le Roux, 2000). A precise hedging scenario for this case is given in Appendix A.

The no-arbitrage GMWB guarantee fees $\alpha_{g}$ for different choices of the mutual fund fees $\alpha_{m}$ (assuming no jumps in $W$ ) are given in Table 6. The table shows that the GMWB fee $\alpha_{g}$ is very sensitive to the mutual fund fee $\alpha_{m}$. The effect of the mutual fund fee on the GMWB fee has not been taken into account previously. Note that a mutual fund fee of $\alpha_{m}=1.0 \%$ increases the GMWB fee by 29 b.p. compared to the case where $\alpha_{m}=0$. The intuition for this is straightforward: the guarantee applies to the initial value of the account $W_{0}$, prior to any fees being deducted. As the mutual fund fees increase, the account value is correspondingly reduced over time, thereby increasing the value of the guarantee.

\subsection{Constant Surrender Charge}

Table 7 provides the GMWB guarantee fees for a constant surrender charge $\kappa=8 \%$ and for the base case $\kappa(t)$ as in Table 1. It is perhaps surprising that the GMWB fee does not appear to 


\begin{tabular}{cc}
\hline & Insurance Fee $\alpha_{g}$ \\
\hline Constant $\kappa$ & 95 b.p. \\
Decreasing $\kappa$ & 117 b.p. \\
\hline
\end{tabular}

TABLE 7: Insurance fees for constant/decreasing $\kappa$. For the decreasing $\kappa(t)$ case, the data is given in Table 1. For the constant $\kappa$ case, the flat rate is 8\%. Other parameter values are in Table 2.

decrease greatly for the case where the surrender fee is constant compared to the case where $\kappa(t)$ decreases to zero. Intuitively, one reason for this is because the reported values are at $t=0$, and the benefit to the investor of the reduced surrender charge is discounted for a relatively lengthy period of time. Moreover, this benefit only arises in states in which it is optimal to withdraw an amount greater than that contractually specified. It is also worth recalling that we are assuming that the surrender charges can be used to fund the guarantee. In general, though, decreasing the fee to zero appears to be mainly a marketing tool, rather than a valuable option for the investor.

\subsection{Sub-optimal Control Strategy}

The previous results were computed assuming an optimal withdrawal policy by the GMWB contract holder. The issue of how to model consumer behaviour when pricing and hedging variable annuities is controversial. It is instructive to reproduce a quote from Cramer et al. (2007):

"Assumptions should reflect that an option will impact policyholder behaviour, and the degree to which it impacts policyholder behaviour will be a function of how much the option is in the money...

Some actuaries believe that all policyholders should be expected to always act optimally, and earnings only recognized when sub-optimal behaviour occurs.

Because the valuation is typically done using risk-neutral assumed returns, some actuaries believe it is appropriate to adjust policyholder behaviour assumptions to reflect policyholder decisions based on a 'real world' environment. Others believe that this approach is inconsistent with a risk neutral framework."

We model non-optimal behavior using the method suggested in Ho et al. (2005). We consider here the sub-optimal behavior of the investor described as follows: at each withdrawal time $\tau^{k}$, the default strategy of the investor is to precisely withdraw the contract withdrawal amount $G^{k}$. Nevertheless, the investor will switch to the optimal withdrawal strategy (if it is different from the default strategy) if the difference between the value corresponding to the optimal strategy and the value corresponding to the default strategy is no less than a fraction $S$ of the initial lump-sum payment. This makes it more likely that the holder will act optimally if the option is deep in the money. To be more precise, the value $V\left(\tau^{k+}\right)$ instantaneously following each withdrawal time $\tau^{k}$ is determined by Algorithm 13.

Effectively, we are assuming that the holder will not bother to withdraw optimally, unless the optimal strategy is considerably more valuable than the default strategy (scaled by the initial lumpsum payment). Table 8 gives the fair insurance fees obtained under the above sub-optimal behavior for different choices of the volatility $\sigma$ and the optimality parameter $S$. We can see from Table 8 that if the holder always withdraws at the default rate, then the value of the guarantee is reduced 


\begin{tabular}{|l|} 
Sub-optimal Behavior \\
$/ /$ Value obtained from the default strategy \\
$U_{d}\left(W, A, \tau^{k+}\right)=V\left(\max \left(W-\gamma_{*}^{k}, 0\right), A-\gamma_{*}^{k}, \tau^{k}\right)+f\left(\gamma_{*}^{k}\right)$, where $\gamma_{*}^{k}=\min \left(A, G^{k}\right)$ \\
$/ /$ Value obtained from the optimal strategy \\
$U_{o}\left(W, A, \tau^{k+}\right)=\sup _{\gamma^{k} \in[0, A]}\left[V\left(\max \left(W-\gamma^{k}, 0\right), A-\gamma^{k}, \tau^{k}\right)+f\left(\gamma^{k}\right)\right]$ \\
$/ /$ Model the sub-optimal behavior by comparing the above two values \\
If $U_{o}\left(W, A, \tau^{k+}\right)-U_{d}\left(W, A, \tau^{k+}\right) \geq S W_{0}$ Then \\
$\quad V\left(W, A, \tau^{k+}\right)=U_{o}\left(W, A, \tau^{k+}\right)$ \\
Else \\
$\quad V\left(W, A, \tau^{k+}\right)=U_{d}\left(W, A, \tau^{k+}\right)$
\end{tabular}

\begin{tabular}{ccc}
\hline Parameter $S$ & $\sigma=.15$ & $\sigma=.20$ \\
\hline 0 (Always withdraw optimally) & 117 b.p. & 214 b.p. \\
0.03 & 86 b.p. & 162 b.p. \\
0.05 & 77 b.p. & 150 b.p. \\
$\infty$ (Always withdraw $G^{k}$ ) & 64 b.p. & 123 b.p. \\
\hline
\end{tabular}

TABLE 8: Insurance fees for the sub-optimal and optimal control strategies. Other parameter values are given in Tables 1 and 2. It is assumed that the holder will take the default action (withdraw at contract rate) unless the gain in acting optimally is larger than $S W_{0}$, where $W_{0}$ is the initial lump-sum payment. See algorithm (13) for details.

by about one-half. However, the sub-optimal GMWB guarantee is still quite valuable for moderate volatilities (123 b.p. for $\sigma=.20)$.

\subsection{Reset Provision}

We next consider a reset provision in the contract. This feature was discussed in Milevsky and Salisbury (2006) and Dai et al. (2007), but no results were reported for this case in those papers. Under this provision, if an excessive withdrawal occurs at some withdrawal time, that is, if $\gamma^{k}>G^{k}$ for some $k$, then the guarantee account balance $A$ is reset to the minimum of $A-\gamma^{k}$ and the resulting sub-account value, $\max \left(W-\gamma^{k}, 0\right)$. Let $\mathcal{A}^{k}$ denote the remaining guarantee account balance after withdrawal. Then we have

$$
\mathcal{A}^{k}=\left\{\begin{array}{ll}
A-\gamma^{k} & \text { if } \gamma^{k} \leq G^{k} \\
\min \left\{A-\gamma^{k}, \max \left(W-\gamma^{k}, 0\right)\right\} & \text { if } \gamma^{k}>G^{k}
\end{array} .\right.
$$




\begin{tabular}{ccc}
\hline & $\sigma=.15$ & $\sigma=.20$ \\
\hline With reset provision & 116 b.p. & 212 b.p. \\
Without reset provision & 117 b.p. & 214 b.p. \\
\hline
\end{tabular}

TABLE 9: Insurance fees with/without reset provision. Other parameter values are given in Tables 1 and 2.

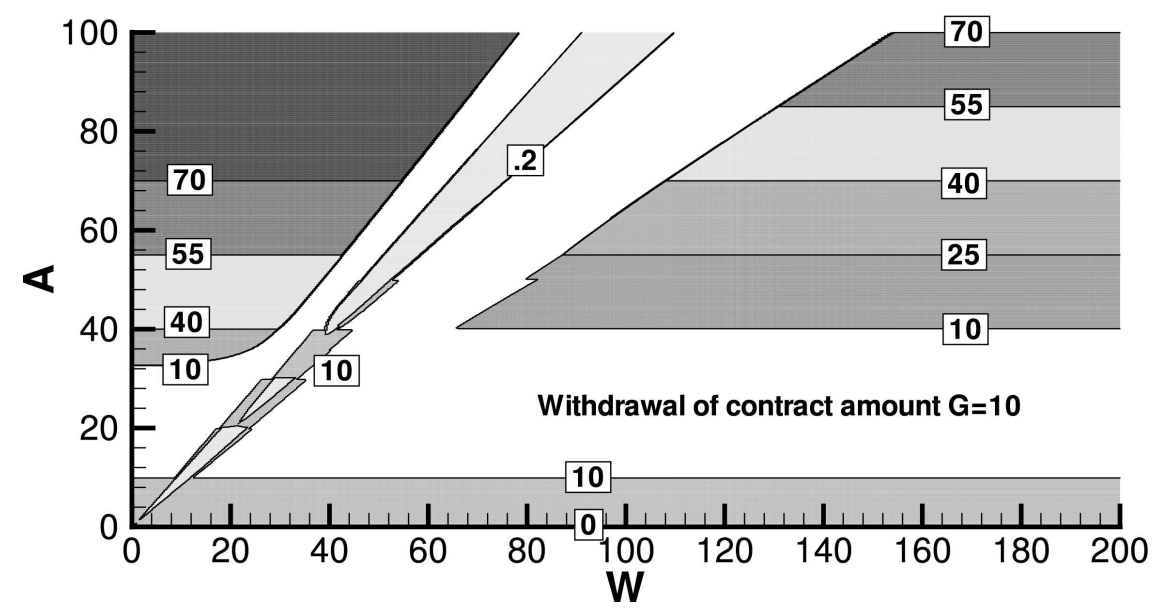

FiguRE 5: Optimal withdrawal strategy of the GMWB guarantee at the first withdrawal time forwards in time $(t=1$ year $)$ in the $(W, A)$-plane with reset provision imposed and with $\sigma=.2$. Other parameters are given in Tables 1 and 2. The contour line showing $\gamma=.2$ shows the region where it is optimal to withdraw essentially nothing.

In this case, the optimality condition (5) becomes

$$
V\left(W, A, \tau^{k+}\right)=\sup _{\gamma^{k} \in[0, A]}\left[V\left(\max \left(W-\gamma^{k}, 0\right), \mathcal{A}^{k}, \tau^{k}\right)+f\left(\gamma^{k}\right)\right], \quad k=0, \ldots, K-1 .
$$

Table 9 provides the fair insurance fees after imposing the reset provision for different choices of volatility. The table shows that the reset provision has little effect on the insurance fee.

Figure 5 shows the optimal withdrawal strategy at the first withdrawal time after imposing reset provision, where we set $\sigma=.20$. Compared with the control strategy without imposing reset provision in Figure 2, we can observe that:

- The right side excessive withdrawal region in Figure 5 is identical to that in Figure 2.

- The left side excessive withdrawal region in Figure 5 amounts to withdrawing all the remaining guarantee account balance, as opposed to the corresponding region in Figure 2, where the remaining balance after excessive withdrawal is 10 . This is due to the extra penalty imposed on $A$ (see equation (14)) that promotes complete withdrawal in order to reduce the resulting loss. Even though the addition of the reset provision (14-15) results in a different optimal strategy when $A \ll W$ compared with the base case, these regions clearly must have a low (risk neutral) probability, so that the guarantee value is almost unchanged (see Table 9). 


\begin{tabular}{cc}
\hline Maturity $T$ & Insurance Fee $\alpha_{g}$ \\
\hline 5 Years & 183 b.p. \\
10 Years & 117 b.p. \\
20 Years & 79 b.p. \\
\hline
\end{tabular}

TABLE 10: Insurance fees for different maturities. Other parameters are given in Tables 1 and 2. The contract withdrawal rate $G^{k}=W_{0} / T$ for these cases.

\begin{tabular}{cc}
\hline Withdrawal Interval & Insurance Fee $\alpha_{g}$ \\
\hline 2 Years & 107 b.p. \\
1 Year & 117 b.p. \\
6 Months & 119 b.p. \\
1 Month & 122 b.p. \\
\hline
\end{tabular}

TABLE 11: Fair insurance fees for different choices of withdrawal intervals. Other parameters are given in Tables 1 and 2. $G^{k}=10 \Delta t_{w}$, where $\Delta t_{w}$ is the withdrawal interval.

\subsection{Different Maturities}

Table 10 gives the fair insurance fees with respect to different maturities. The contract withdrawal rate $G^{k}=W_{0} / T$ for these cases. As might be expected, the value of the guarantee decreases as the maturity increases, due to the reduced time value of the guarantee (recall that the total guaranteed withdrawal amount initially is $W_{0}$, irrespective of the maturity date).

\subsection{Different Withdrawal Intervals}

Table 11 gives the fair insurance fees with respect to different withdrawal intervals. In this case, the maximum withdrawal without penalty is given by $G^{k}=10 \Delta t_{w}$, where $\Delta t_{w}$ is the withdrawal interval. The table shows that the withdrawal intervals have a fairly small effect on the insurance fees. For example, decreasing the withdrawal interval from one year to one month increases the fee by only 5 b.p. This means that allowing more frequent withdrawals (but at the same yearly rate) does not increase the value of the guarantee significantly.

\subsection{Varying Interest Rates}

Table 12 gives the fair insurance fees with respect to different values of the risk free interest rate $r$. The guarantee values are extremely sensitive to interest rates, due to the time value of the guarantee account. A reduction in the risk free rate drastically increases the value of the GMWB guarantee.

\section{Conclusions}

We have carried out an extensive analysis of the no-arbitrage fee for GMWB guarantees. Typical fees for GMWB guarantees are less than 50 b.p. (Milevsky and Salisbury, 2006). Fees of this level 


\begin{tabular}{cc}
\hline Interest Rate & Insurance Fee $\alpha_{g}$ \\
\hline $1 \%$ & 761 b.p. \\
$3 \%$ & 227 b.p. \\
$5 \%$ & 117 b.p. \\
$7 \%$ & 68 b.p. \\
$9 \%$ & 41 b.p. \\
\hline
\end{tabular}

TABLE 12: Fair insurance fees for different values of $r$. Other parameters are given in Tables 1 and 2.

can be justified (assuming that the guarantee is hedged) only if all of the following assumptions are made:

- Volatilities are $15 \%$ or less. Market crashes (jumps) are either impossible or at least extremely unlikely over the lifetime of these contracts (under the pricing measure).

- Contract holders withdraw sub-optimally.

- Interest rates do not drop to relatively low levels.

In all other cases, the GMWB no-arbitrage fee should be considerably higher than 50 b.p. This would suggest that insurers are exposed to considerable risk, since the fees collected are not enough to cover hedging costs.

In many cases, the business unit hedging the guarantee is separate from the unit managing the mutual fund. For typical parameters, the GMWB hedging fee increases by about 30 b.p. due to this fee splitting.

We note that most GMWB contracts have additional optionality (e.g. ratchet provisions and increases in the guarantee account if withdrawals are skipped), which can only increase the value of the guarantee. Finally, we have implicitly assumed throughout this paper (this is made explicit in Appendix A) that the insurer can hedge these contractual obligations using a traded instrument that is perfectly correlated with the investment in the investor's account. In other words, we have ignored basis risk. Of course, our stochastic modelling assumptions are also rather simple (e.g. constant volatility and interest rates). Hedging risk exposures arising from such real world features would require additional reserves. Consequently, the guarantee values calculated in this paper will undervalue typical contracts. This reinforces our primary conclusion that insurance companies are not charging enough to hedge these contracts.

\section{A Derivation of Contract Equations}

In this Appendix, we will derive the equations (4), (5) and (6) for the annuity value. In many cases, the GMWB guarantee is provided as a separate rider for an underlying investment in a mutual fund. The GMWB rider is funded by a fee which is distinct from any management fees associated with the mutual fund. The mutual fund management may be a completely separate business unit from the unit providing the GMWB guarantee, hence the mutual fund management fees are not available to fund the GMWB guarantee. Under this scenario, the unit managing 
the guarantee is not permitted to short the mutual fund, and uses an index proxy to hedge the guarantee (Le Roux, 2000). Therefore, it is important to distinguish between these two sets of fees.

We will first derive the equations for the pure GMWB guarantee. We will use the same arguments used in Windcliff et al. (2001) to model segregated funds. We then convert these equations into the value of the total variable annuity, which can then be related to previous work on GMWB guarantees (Milevsky and Salisbury, 2006; Dai et al., 2007).

Let $\alpha_{m}$ be the proportional fee charged by the manager of the underlying mutual fund. Let $\alpha_{g}$ be the fee used to fund the GMWB guarantee, with $\alpha_{t o t}=\alpha_{m}+\alpha_{g}$. For simplicity, we will derive the equations assuming that the underlying asset follows Geometric Brownian Motion.

Consider the following scenario. The underlying asset $W$ in the investor's account follows

$$
d W=\left(\mu-\alpha_{t o t}\right) W d t+W \sigma d Z
$$

where $\mu$ is the drift rate and $d Z$ is the increment of a Wiener process. We ignore withdrawals from the account in equation (16) for the moment. We assume that the mutual fund tracks an index $\hat{W}$ which follows the process

$$
d \hat{W}=\mu \hat{W} d t+\hat{W} \sigma d Z .
$$

We assume that it is not possible to short the mutual fund, so that the obvious arbitrage opportunity cannot be exploited. We further assume that it is possible to track the index $\hat{W}$ without basis risk.

Now, consider the writer of the GMWB guarantee, with no-arbitrage value $U(W, A, t)$. The terminal condition is

$$
U(W, A, t=T)=\max (A(1-\kappa)-W, 0)
$$

which represents the cashflow which must be paid by the guarantee provider. The writer sets up the hedging portfolio

$$
\Pi(W, \hat{W}, t)=-U(W, t)+x \hat{W},
$$

where $x$ is the number of units of the index $\hat{W}$.

Over the time interval $t \rightarrow t+d t$, between withdrawal dates,

$$
\begin{aligned}
d \Pi & =-\left[\left(U_{t}+\left(\mu-\alpha_{t o t}\right) W U_{W}+\frac{1}{2} \sigma^{2} W^{2} U_{W W}\right) d t+\sigma W U_{W} d Z\right] \\
& +x[\mu \hat{W} d t+\sigma \hat{W} d Z]+\alpha_{g} W d t
\end{aligned}
$$

where the term $\left(\alpha_{g} W d t\right)$ represents the GMWB fee collected by the hedger. Choose

$$
x=\frac{W}{\hat{W}} U_{W}
$$

so that equation (20) becomes

$$
d \Pi=-\left[\left(U_{t}-\alpha_{t o t} W U_{W}+\frac{1}{2} \sigma^{2} W^{2} U_{W W}\right) d t\right]+\alpha_{g} W d t .
$$

Setting $d \Pi=r \Pi d t$ (since the portfolio is now riskless) gives

$$
U_{\tau}=\frac{1}{2} \sigma^{2} W^{2} U_{W W}+\left(r-\alpha_{t o t}\right) W U_{W}-r U-\alpha_{g} W,
$$


where $\tau=T-t$. Note that equation (23) has the same form as that used to value segregated fund guarantees (Windcliff et al., 2001, 2002).

At withdrawal times $\tau^{k}$, the holder of the GMWB will maximize the value of the guarantee, so that

$$
U\left(W, A, \tau^{k+}\right)=\sup _{\gamma^{k} \in[0, A]}\left[U\left(\max \left(W-\gamma^{k}, 0\right), A-\gamma^{k}, \tau^{k}\right)+f\left(\gamma^{k}\right)-\min \left(\gamma^{k}, W\right)\right] .
$$

Note that

$$
f\left(\gamma^{k}\right)-\min \left(\gamma^{k}, W\right)=\left\{\begin{array}{ll}
0 & \gamma^{k} \leq G^{k} ; \gamma^{k}<W \\
-\kappa\left(\gamma^{k}-G^{k}\right) & \gamma^{k}>G^{k} ; \gamma^{k}<W \\
\gamma^{k}-W & \gamma^{k} \leq G^{k} ; \gamma^{k}>W \\
\left(\gamma^{k}-W\right)-\kappa\left(\gamma^{k}-G^{k}\right) & \gamma^{k}>G^{k} ; \gamma^{k}>W
\end{array},\right.
$$

which represents the total cash outflows from the writer of the guarantee to the holder of the GMWB contract, i.e. cashflows required to make up any guarantee shortfall net of penalties for withdrawals above the contract amount.

Now, let $V(W, A, \tau)$ be the value of the total variable annuity contract, i.e.

$$
V=U+W
$$

In other words, $V$ is the total variable annuity value, which includes the amount in the risky account and the separate GMWB guarantee. Substituting equation (26) into equation (18) gives

$$
V(W, A, \tau=0)=\max (W, A(1-\kappa)) .
$$

Similarly, substituting (26) into (23) gives

$$
V_{\tau}=\frac{1}{2} \sigma^{2} W^{2} V_{W W}+\left(r-\alpha_{t o t}\right) W V_{W}-r V+\alpha_{m} W
$$

and finally, at withdrawal times $\tau^{k}$ we obtain (from equations (26) and (24))

$$
V\left(W, A, \tau^{k+}\right)=\sup _{\gamma^{k} \in[0, A]}\left[V\left(\max \left(W-\gamma^{k}, 0\right), A-\gamma^{k}, \tau^{k}\right)+f\left(\gamma^{k}\right)\right] .
$$

Note that if $\alpha_{m}=0$, then equations (27 - 29) reduce to the GMWB equations derived in Dai et al. (2007) for the discrete withdrawal case.

Although at first sight the term $\alpha_{m} W$ on the right hand side of equation (28) seems counterintuitive, we can also derive this equation assuming the following scenario. Imagine that the hedger replicates the cash flows associated with the total GMWB contract. In this case, the underlying mutual fund can be regarded as a purely virtual instrument, following process (16). The hedging instrument follows process (17), and the hedging unit pays a sales fee of $\alpha_{m} W$ to the mutual fund unit. In other words, rather than having the investor directly pay the proportional fees $\alpha_{m}$ to the mutual fund unit and $\alpha_{g}$ to the guarantee provider, the investor pays a proportional fee of $\alpha_{t o t}=\alpha_{m}+\alpha_{g}$ to the guarantee provider, which keeps $\alpha_{g} W$ to hedge the guarantee and passes along $\alpha_{m} W$ to the mutual fund unit. Following a similar argument as in equations (19-23), and noting that the hedger must pay the mutual fund fees $\alpha_{m} W$, results in equation (28). 


\section{References}

Andersen, L. and J. Andreasen (2000). Jump-diffusion processes: Volatility smile fitting and numerical methods for option pricing. Review of Derivatives Research 4, 231-262.

Bauer, D., A. Kling, and J. Russ (2006). A universal pricing framework for guaranteed minimum benefits in variable annuities. Working paper, Ulm University.

Chen, Z. and P. Forsyth (2007). A numerical scheme for the impulse control formulation for pricing variable annuities with a Guaranteed Minimum Withdrawal Benefit (GMWB). Submitted to Numerische Mathematik.

Cramer, E., P. Matson, and L. Rubin (2007). Common practices relating to FASB statement 133, Accounting for Derivative Instruments and Hedging Activities as it Relates to Variable Annuities with Guaranteed Benefits. Practice Note, American Academy of Actuaries.

Dai, M., Y. K. Kwok, and J. Zong (2007). Guaranteed minimum withdrawal benefit in variable annuities. Mathematical Finance, forthcoming.

d'Halluin, Y., P. Forsyth, and K. Vetzal (2005). Robust numerical methods for contingent claims under jump diffusion processes. IMA Journal of Numerical Analysis 25, 65-92.

He, C., J. Kennedy, T. Coleman, P. Forsyth, Y. Li, and K. Vetzal (2006). Calibration and hedging under jump diffusion. Review of Derivatives Research 9, 1-35.

Ho, T., S. B. Lee, and Y. Choi (2005). Practical considerations in managing variable annuities. Working paper, Thomas Ho Company.

Kennedy, J., P. Forsyth, and K. Vetzal (2006). Dynamic hedging under jump diffusion with transaction costs. Submitted to Operations Research.

Le Roux, M. (2000). Private communication. Director of product development, ING Institutional Markets.

Milevsky, M. A. and T. S. Salisbury (2006). Financial valuation of guaranteed minimum withdrawal benefits. Insurance: Mathematics and Economics 38, 21-38.

Windcliff, H., P. Forsyth, M. Le Roux, and K. Vetzal (2002). Understanding the behaviour and hedging of segregated funds offering the reset feature. North American Actuarial Journal 6, $107-125$.

Windcliff, H., P. Forsyth, and K. Vetzal (2001). Valuation of segregated funds: shout options with maturity extensions. Insurance: Mathematics and Economics 29, 1-21. 